\title{
Strange baryons in a hot and dense medium within the Nambu-Jona-Lasinio model
}

\author{
F. Gastineau and J. Aichelin \\ SUBATECH \\ Laboratoire de Physique Subatomique et des Technologies Associées \\ UMR Université de Nantes, IN2P3/CNRS, Ecole des Mines de Nantes \\ 4, rue Alfred Kastler, F-44070 Nantes Cedex 03, France.
}

\begin{abstract}
Using an extended version of the Nambu-Jona-Lasinio model we build a simple description of the baryons as diquark-quark bound states. First, a description of the diquarks in a dense and hot medium is presented. Then, we introduce the formalism for the baryons based on the Faddeev equation associated with the so-called "static approximation" which finally gives a Bethe-Salpeter equation in the diquarks-quarks channel. By identifying the baryons with the bound states, we can obtain a description of their properties. In particular, we obtain the right mass spectrum for the proton, $\Lambda, \Xi$, and $\Sigma$ at $\mathrm{T}=0$ and $\mu=0$. We extend the formalism to finite temperature and density to obtain a description of the mass change of these baryons in the medium. proc
\end{abstract}




\section{Introduction}

Knowing the properties of hadrons in a hadronic environment is presently one of the challenging problems in heavy ion physics. Experimentally, the first results have been published with clear evidence that the properties of mesons change considerable when they are placed into a hadronic medium [1]. Theoretically, most of the efforts are concentrated around the mesons, where a substantial change of their rest mass is predicted. Baryons have attracted less attention. However, the knowledge of their properties at finite density and temperature is a prerequisite for interpreting the experimental results of heavy ion collisions at ultra-relativistic energies. There, it is assumed that a plasma of quarks and gluons is formed during the reaction. This plasma then expands and cools. When it passes the critical temperature for the chiral and deconfined phase transitions, it hadronizes into a colorless gas of hadrons. Since baryon masses change much more as a function of the density as compared to meson masses, the thermodynamic properties of the hadron gas and especially the particle abundances at this transition will be strongly influenced by the baryon properties in

a medium. The Nambu-Jona-Lasinio(NJL) model offers the possibility to determine the properties of the baryons at finite temperature and density. In this model the baryons are described as diquark-quark bound states.

\section{The NJL model}

The NJL model provides a simple implementation of dynamically broken chiral symmetry. It has been successfully used for the description of mesonic states at lowenergy [2] and some work has been done for baryons at zero temperature and density [3, 4, 5. We use an SU(3) version of the NJL Lagrangian adding some extra terms for the qq channel interaction:

$$
\begin{aligned}
\mathcal{L}_{\bar{q} q} & =G_{S}\left[\left(\bar{\psi} \lambda^{F} \psi\right)^{2}+\left(\bar{\psi} i \gamma^{5} \lambda^{F} \psi\right)^{2}\right]+G_{V}\left[\left(\bar{\psi} \lambda^{F} \gamma_{\mu} \psi\right)^{2}+\left(\bar{\psi} i \gamma^{5} \gamma_{\mu} \lambda^{F} \psi\right)^{2}\right] \\
& +G_{D}\left[\operatorname{det} \bar{\psi}\left(1+\gamma^{5}\right) \psi+\bar{\psi}\left(1-\gamma^{5}\right) \psi\right] \\
\mathcal{L}_{q q} & =G_{D I Q}\left[\left(\bar{\psi}^{c} i \gamma_{5} \lambda_{F}^{A} \lambda_{C}^{A^{\prime}} \psi\right)\left(\bar{\psi} i \gamma^{5} \lambda_{F}^{A} \lambda_{C}^{A^{\prime}} \psi^{c}\right)\right]
\end{aligned}
$$

where $A, A^{\prime}=2,5,7$ projects on the color and flavor $\overline{3}$ channel.

The ratio $G_{S} / G_{D I Q}$ is in principle fixed by the Fierz transformation, but we choose to leave it as a free parameter. The model needs to be regularized with a 3 -momentum cut-off $\Lambda$. The parameters are fixed in order to give correct value for the pion decay constant, quark condensate, mesons masses. In this work we have used the follow ing parameters: $m_{0, q}=3.95 \mathrm{MeV}, m_{0, s}=148 \mathrm{MeV}, G_{S} / \Lambda^{2}=1.92 \mathrm{MeV}^{-2}$, $G_{D} / \Lambda^{2}=10.0 \mathrm{MeV}^{-2}, G_{V} / \Lambda^{2}=3.55 \mathrm{MeV}^{-2}, \Lambda=708 \mathrm{MeV}, G_{D I Q} / G_{S}=0.73$

\section{The diquarks}

Before describing the baryonic states, we must study the behavior of diquarks. They are (q-q) bound states, and therefore not observable in nature do their lack of color neutrality. First, we determine their mass in a hot and dense medium using the same formalism as for the mesons [2, 6], the Bethe-Salpeter equation. In the qq channel it looks like :

$$
\mathcal{T}\left(q^{2}\right)=\mathcal{K}+\frac{i}{2} \int \frac{d^{4} p}{(2 \pi)^{4}}\left[\mathcal{K} S_{F}(p+q) S_{F}(-q) \mathcal{T}\left(q^{2}\right)\right]
$$




\begin{tabular}{llll}
\hline & $\xi$ & & \\
\hline & & 6 \\
\hline
\end{tabular}

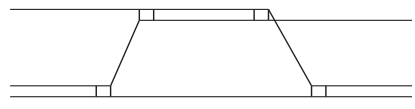

Figure 1. Simple picture of the representation of baryons in our NJL model. Double lines are diquarks where as simple lines are quarks

where $\mathcal{K}=\Omega^{a} 2 i G_{D I Q} \bar{\Omega}^{b}\left(a, b\right.$ describe diquarks) with $\Omega^{i}=\left(\lambda_{C}^{B} \otimes \lambda_{F}^{A} \otimes \Gamma^{i} C\right)$. Here, we limit ourselves to the scalar channel $\left(\Gamma^{i}=i \gamma^{5}\right)$. The axial contribution to the baryon, which is not negligible as already shown in 4, will be treated later.

In the NJL model RPA and Bethe-Salpeter equation are identical and can be rewritten as a geometric series. Therefore one finally gets a simple form for the Tmatrix, where the singularities determine the bound states. In order to determine their mass one need to solve the gap equation: $\operatorname{det}\left(1+\Pi\left(q^{2}\right) K\right)=0$ for $m_{d}^{2}=q^{2}$ where $m_{d}$ is the diquark mass. We have introduced here the polarization function defined by : $\Pi^{i j}\left(q^{2}\right)=-\int^{\Lambda} \frac{d^{4} q}{(2 \pi)^{4}}\left[\bar{\Omega}_{\alpha^{\prime} \beta^{\prime}}^{i} S_{F}^{\alpha^{\prime} \delta^{\prime}}(p+q) S_{F}^{\beta^{\prime} \gamma^{\prime}}(-p) \bar{\Omega}_{\delta^{\prime} \gamma^{\prime}}^{j}\right]$

Previous calculations have been done in [3] for $T=0$ and $\mu=0$.

To extend this equation to finite temperature and density, we used the imaginary time formalism as described in [7]. In order to build baryon states we need the coupling constant between a diquark and two quarks. It can be defined by $g^{-2} \propto$ $\left.\left(\partial \Pi\left(k^{2}\right) / \partial k^{2}\right)\right|_{k^{2}=m_{d}^{2}}$.

\section{Baryons as diquarks-quarks bound state}

The basic idea is to build baryons in the same way as mesons or diquarks, using the Bethe-Salpeter equation. To do this we need to know the diquark-quark interaction vertex which is not given in the Lagrangian. Therefore we have to add it in an empirical way. We start from the basic picture of a baryon (3 quarks interacting through gluon exchange) and apply it to the NJL model. Each interaction is pointlike, and two interacting quarks form a bound state (Fig. 1). The result is that quarks and diquarks interact by exchanging quarks. Following ref. [4] we assume that the mass of the exchanged quark is infinite ("static approximation"). and therefore the interaction becomes point-like, with the vertex: $\mathcal{Z}_{b c}^{\beta \gamma}=g_{D q q^{\prime}}^{\prime} \Omega_{a}^{\beta \delta}\left(-i / M_{e x}\right) \bar{\Omega}_{b}^{\gamma \alpha} g_{D q q^{\prime}}$. The Bethe-Salpeter equation for baryons reads:

$$
\mathcal{T}_{b a}^{\beta \alpha}(\mathbf{P})=\mathcal{Z}_{b a}^{\beta \alpha}+\int \frac{d^{4} \mathbf{k}}{(2 \pi)^{4}} \mathcal{Z}_{b c}^{\beta \gamma} S_{F l}^{\gamma \delta}(k) S_{D, c d}^{l}(P-k) \mathcal{T}_{d a}^{\delta \alpha}(\mathbf{P}),
$$

where we have omitted the sum over the exchanged quarks, which is present if we study $\Lambda$ (uds). Instead of a quark and an anti-quark propagator we have now the product of different propagators: one for a quark and one for a diquark. Following the usual approach to get the gap equation for baryons

$$
\begin{aligned}
& \operatorname{det}(1-\Pi Z)=0 \\
& \text { where } \Pi\left(P^{2}\right)=-\int \frac{d^{4} \mathbf{p}}{(2 \pi)^{4}} T_{i} \bar{\Omega}_{c}^{\alpha \delta} i S_{F}^{\delta \gamma}(p) i S_{D}^{c d}(P-p) \Omega_{d}^{\gamma \beta} T_{j}
\end{aligned}
$$

for $P^{2}=m_{B}^{2} . T_{i}, T_{j}$ are the isospin projections on the baryonic state. The finite temperature and density are taken into account using the imaginary time formalism. 


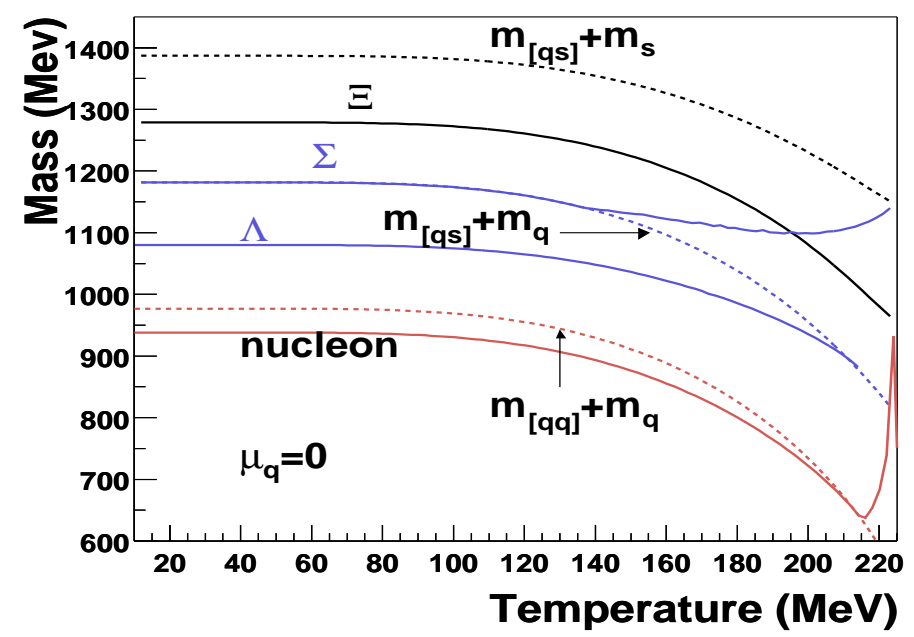

Figure 2. Temperature dependence of the baryon masses (solid line). The dashed line represents the sum of the two baryon's constituent masses(diquark + quark).

\section{Results}

The parameters of Lagrangian (11) lead to a cross-over phase transition in the whole $(T, \mu)$ plane. Along the T-axis, $m_{q}(T) / m_{q}(T=0)=1 / 2$ for $T=220 \mathrm{MeV}$. For the $\mu$-axis, $m_{q}(\mu) / m_{q}(\mu=0)=1 / 2$ for $\rho / \rho_{0}=2.5$ and $T_{c}=220 \mathrm{MeV}$.

At $T=\mu=0$ all the particles are stable with masses: $m_{q}=422 \mathrm{MeV}, m_{s}=628$ $\mathrm{MeV}, m_{q q}=557 \mathrm{MeV}, m_{q s}=760 \mathrm{MeV}, m_{\text {nucleon }}=938 \mathrm{MeV}, m_{\Lambda}=1080 \mathrm{MeV}$, $m_{\Sigma}=1181 \mathrm{MeV}$ and $m_{\Xi}=1278 \mathrm{MeV}$.

In Fig. 2, we display the mass of baryons as a function of the temperature. All masses decrease with increasing temperature. This mass change is due to chiral symmetry restoration $\left(m_{q}, m_{s}\right.$ go to their bare masses). In contradiction to models which have baryons or mesons as degrees of freedom, the instability of baryons with respect to diquark-quark system (dashed line in Fig. 2) can be calculated. Nucleons and $\Lambda$ get unstable already at $T \approx 220 \mathrm{MeV}$, where as the $\Xi$ is stable longer due to its large strangeness content(s quark masses do not change too much with T). $\Sigma$ is a special case due to its weak binding energy, when the quark mass changes $\Sigma$ becomes unstable.

In Fig. 3 we plot the masses of baryons as function of the density for $\mathrm{T}=0$. As we observed in fig 2, all masses decrease and baryons become unstable for a given density $\left(\rho / \rho_{0} \approx 2.6\right.$ for the nucleon $\left(M_{p, n} \approx 425 \mathrm{MeV}\right)$ and $\Lambda\left(M_{\Lambda} \approx 850 \mathrm{MeV}\right) ; \rho / \rho_{0} \approx 1.7$ for $\Sigma\left(M_{\Sigma} \approx 950 \mathrm{MeV}\right)$. The value of the nucleon mass at $\rho / \rho_{0}=1$ is about $700 \mathrm{MeV}$. This is in agreement with the results of Walecka or HQT models [8] and also follow the results obtained in the other approaches [9, 10] with the exception that in the NJL approaches we can also predict when baryons become unstable against description into a q - (qq) state. 


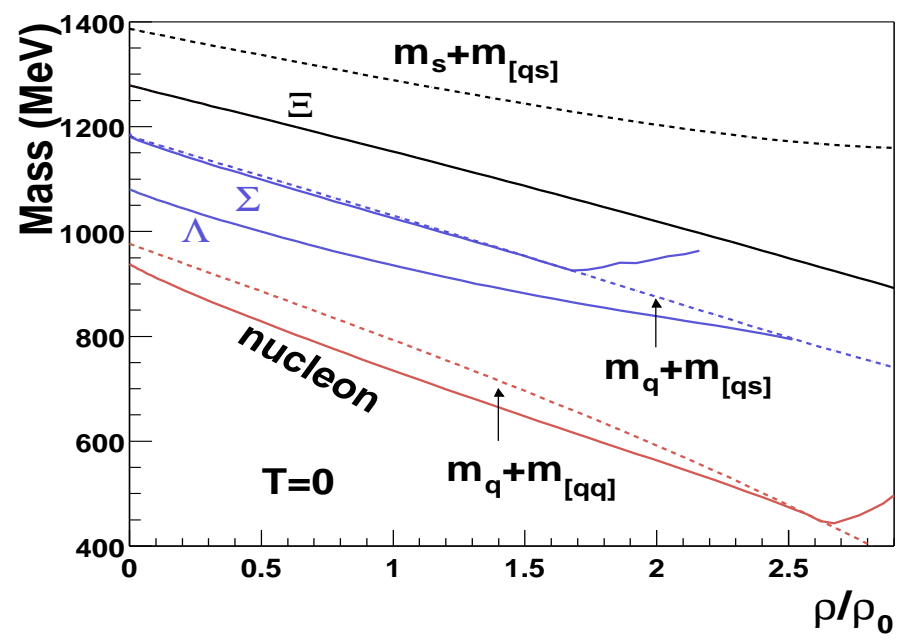

Figure 3. Density dependence of the nucleon, $\Sigma, \Lambda, \Xi$ masses are shown(solid lines) and upper limit for the baryon masses(dashed lines): the sum of the quark and diquark masses for each particle.

\section{Conclusion}

Employing the Nambu-Jona-Lasinio model we have constructed a simple formalism to described baryons as diquark-quark bound state. Extending the gap equation to finite temperature and density, we show that baryon masses decrease by almost $30 \%$ close the chiral phase transition as compared to the vacuum values. This mass change may affect baryon multiplicity when a plasma hadronises into a hadron gas. It can also modify the thermal freeze-out point as already shown in [11]. The next challenge is to describe the phase transition from a quark, diquark plasma into a hadron gas. A first step in this direction has been done for the mesons [12], but not yet for the baryons. Our simple model can easily be employed to study the phase transition at finite baryon density.

[1] Quark Matter 2001 Nucl. Phys. A698 (2002)

[2] S.Klimt, M. Lutz, U. Vogl and W. Weise Nucl. Phys. A516 (1990) 429-468, S. P Klevansky Rev. Mod. Phys. Vol 64, No.3 July 1992.

[3] N. Ishii, W.Bentz, K. Yazaki Nucl. Phys. A 587(1995) 617-656

[4] A. Buck, R. Alkofer and H. Reinhardt Phys. Lett. B 286 (1992) 29-35

[5] S. Huang, J. Tjon Phys. Rev. C 49,1702 (1994)

[6] P. Rehberg and al. Phys. Rev. C 53 (1996) 410-429

[7] J. I. Kapusta, Finite-Temperature field Theory Cambridge University Press(1989)

[8] B.D. Serot, J. D. Walecka Int.J.Mod.Phvs.E6:515-631,1997

[9] W. Bentz and A.E. Thomas nucl-th/0105022

[10] P. Papazoglou, D. Zschiesche, S. Schramm, H. Stocker, W. Greiner J. Phys. G: Nucl. Part. Phys. 23:2081-2089,1997

[11] W. Florkowski, W. Broniowski Phys. Lett. B 477 (2000) 73-76

[12] P. Rehberg, J. Aichelin Phys.Rev.C 60:064905,1999 